\title{
Quantum and Classical Approach Applied to the Motion of a Celestial Body in the Solar System
}

\author{
Stanisław Olszewski \\ Institute of Physical Chemistry, Polish Academy of Sciences, Warsaw, Poland \\ Email: olsz@ichf.edu.pl
}

How to cite this paper: Olszewski, S. (2016) Quantum and Classical Approach Applied to the Motion of a Celestial Body in the Solar System. Journal of Modern Physics, 7, 1901-1908.

http://dx.doi.org/10.4236/jmp.2016.714168

Received: September 22, 2016

Accepted: October 15, 2016

Published: October 19, 2016

Copyright (c) 2016 by author and Scientific Research Publishing Inc. This work is licensed under the Creative Commons Attribution International License (CC BY 4.0).

http://creativecommons.org/licenses/by/4.0/

\begin{abstract}
According to the classical mechanics the energy of a celestial body circulating in the solar system is a constant term. This energy is defined by the masses product of the larger and smaller body entering into a mutual attraction as well as the size of the major semiaxis characteristic for the corresponding Kepler orbit. A special situation concerns the planet interaction with the Sun because of a systematic decrease of the Sun mass due to the luminosity effect. The aim of the paper is to point out that even in the case of perfectly constant interacting masses the energy of the moving body should decrease when a quantum treatment of the body motion is considered. The rate of the energy decrease is extremely small, nevertheless it gives a shortening of the distance between the interacting bodies leading to a final effect of a touch of the larger body and a smaller one.
\end{abstract}

\section{Keywords}

Solar System, Classical Mechanics and Quantum Theory, Emission Rate of Energy by a Moving Planet, The Earth Planet Taken as an Example

\section{Introduction}

A selection of physical objects to the quantum or classical kind of a study is well known. In principle the microscopic bodies representing mainly the atomic and molecular systems are the objects belonging to the quantum kind of approach. On the other side there do exist the celestial bodies for which the classical treatment of their motion is a well-established theory.

Paradoxally, in spite of a different kind of forces and an extremely different size of the geometrical parameters entering the microscopic and celestial physical world, respectively, the mathematical treatment developed in the Bohr atomic theory and Newton mechanics of the solar system are much similar [1] [2]. 
The energy of the moving body-which is an electron in the hydrogen atom and a planet or satellite in the solar system-is a constant number, and the same property concerns the angular momentum. In fact both theories represent the two-body problem of the interaction between a stable heavy body with a much less heavy moving body. An essential difference between the atomic and solar systems is that a stationary electron energy in an atom-excepting for the situation characteristic for the atomic ground state-can be spontaneously changed, but it seems that no similar change can apply to the energy of a planet or satellite.

The aim of the present paper is to examine a possibility of a spontaneous change of the constant energy attributed to a celestial body moving in the solar system.

To the best knowledge of the author this problem has been never investigated before and no similar study has been raised in the past.

\section{Quantization of the Motion of a Celestial Body}

If the trajectory of the body is a definite closed path, the old quantum theory refers the quantum number $n$ of the body state to its momentum $\boldsymbol{p}$ by the formula [1]

$$
S=\oint \boldsymbol{p} \mathrm{d} \boldsymbol{r}=\oint m v \mathrm{~d} \boldsymbol{r}=(n+1) h ;
$$

the index $n+1$ instead of $n$ has been introduced for the sake of convenience, $h$ is the Planck constant. In principle there is no limit for the integer number $n$.

If the path can be approximated by a circle of radius $r$ and the body velocity $|v|=v$ on the circle is roughly constant, we obtain for (1) the equation

$$
S \cong 2 \pi m v r=(n+1) h \text {; }
$$

$m$ is the mass of the body.

Henceforth let us specialize the calculations to the motion of the Earth planet taken as an example. In fact no essential difference does concern the Earth case and cases represented by other planets or satellites interacting with their gravitational centers, on condition the luminosity effect of the mass decrease of the Sun in neglected [5]. We have for the Earth planet [3] [4] the mass

$$
m=5.976 \times 10^{24} \mathrm{~kg} ;
$$

the average velocity on the Kepler orbit

$$
v=29.79 \mathrm{~km} \cdot \mathrm{s}^{-1},
$$

and the average distance from the Sun

$$
r=149.6 \times 10^{6} \mathrm{~km} \text {. }
$$

With the data in (3)-(5) and

$$
h=6.62 \times 10^{-27} \mathrm{erg} \cdot \mathrm{sec}
$$

the Formula (2) becomes

$$
S \cong 2 \pi m_{E} v_{E} r_{E}^{(n+1)}=(n+1) h \cong 4 \times 10^{73} h .
$$

In the sense of the quantum theory the Earth is on a high quantum level 


$$
n+1=4 \times 10^{73}
$$

With $S$ given in (7) there is connected the energy [6]

$$
E_{n+1}=-\frac{G M m}{2 a_{n+1}} \cong-\frac{G M m}{2 r_{n+1}}
$$

where $a_{n+1}$ denotes a larger semiaxis of the Kepler orbit, $r$ (with subscript $n+1$ ) replaces $r$ presented in (5),

$$
G \cong 6.67 \times 10^{-8}
$$

is the gravitational constant when the masses in (9) are in grams and the distances in centimeters [5], and [3] [4]

$$
M=1.99 \times 10^{30} \mathrm{~kg}
$$

is the mass of the Sun.

\section{Spontaneous Emission of Energy by a Quantum System Applied to the Case of a Moving Earth}

A quantum system being in the state $n+1$ can spontaneously emit its energy by going to a lower state $n$. In this case $S$ in (7) is changed (decreased) by the interval

$$
\begin{aligned}
\Delta S & =(n+1-n) h=h=2 \pi m\left[(v r)_{n+1}-(v r)_{n}\right] \\
& =2 \pi m\left[\left(v_{n}+\Delta v_{n}\right)\left(r_{n}+\Delta r_{n}\right)-v_{n} r_{n}\right] \cong 2 \pi m\left(\Delta v_{n} r_{n}+v_{n} \Delta r_{n}\right)
\end{aligned}
$$

where

$$
\begin{aligned}
\Delta v_{n} & =v_{n+1}-v_{n}, \\
\Delta r_{n} & =r_{n+1}-r_{n} .
\end{aligned}
$$

Respectively the energy is changed (decreased) by

$$
\Delta E=E_{n+1}-E_{n} \cong-\frac{G M m}{2}\left(\frac{1}{r_{n+1}}-\frac{1}{r_{n}}\right)=-\frac{G M m}{2}\left(r_{n}-r_{n+1}\right) \frac{1}{r_{n+1} r_{n}} \cong \frac{G M m}{2 r_{n}^{2}} \Delta r_{n} .
$$

The $\Delta E$ is a positive number because

$$
r_{n}<r_{n+1} .
$$

Finally due to the virial theorem [7] valid for any $n$ we have

$$
2 \bar{E}_{\mathrm{kin}}^{(n)}+\bar{E}_{\mathrm{pot}}^{(n)}=E_{n}+\bar{E}_{\mathrm{kin}}^{(n)}=0 .
$$

Here $\bar{E}_{\text {kin }}^{(n)}$ and $\bar{E}_{\text {pot }}^{(n)}$ are respectively the kinetic and potential energy averaged over the Kepler orbit, therefore

$$
E_{n}=-\bar{E}_{\mathrm{kin}}^{(n)}
$$

In effect of another representation of $\Delta E$ than (13), the formula

$$
\Delta E=-\frac{m}{2}\left(v_{n+1}^{2}-v_{n}^{2}\right)=-\frac{m}{2}\left(v_{n+1}-v_{n}\right)\left(v_{n+1}+v_{n}\right) \cong-\frac{m}{2} \Delta v_{n} 2 v_{n}=-m \Delta v_{n} v_{n},
$$

is also valid. As a result we obtain three Equations [(12), (13) and (16)] for three 
unknown parameters

$$
\Delta r, \Delta v \text { and } \Delta E \text {. }
$$

The equations can be easily solved by putting in the first step the $\Delta r_{n}$ calculated from (13) and $\Delta v_{n}$ from (16) into (12):

$$
h \cong 2 \pi m\left(-\frac{\Delta E}{m v_{n}} r_{n}+v_{n} \frac{2 \Delta E r_{n}^{2}}{G M m}\right)
$$

This gives

$$
\Delta E=\frac{h}{2 \pi m}\left(-\frac{r_{n}}{m v_{n}}+\frac{2 v_{n} r_{n}^{2}}{G M m}\right)^{-1}=\frac{\hbar}{r_{n}}\left(-\frac{1}{v_{n}}+\frac{2 v_{n} r_{n}}{G M}\right)^{-1} \cong \frac{\hbar}{r_{n}}\left(-\frac{1}{v_{n}}+\frac{2}{v_{n}}\right)^{-1}=\frac{\hbar v_{n}}{r_{n}} .
$$

In obtaining the Formula (19) the approximate relation

$$
\frac{1}{v_{n}} \cong \frac{r_{n} v_{n}}{G M}
$$

has been applied. The (20) is descending from the ratio of the kinetic energy of the body to the absolute value of the body energy represented by the approximate Formula (9):

$$
\frac{E_{\text {kin }}^{(n)}}{\left|E_{n}\right|} \cong \frac{\frac{m v_{n}^{2}}{2}}{\frac{G M m}{2 r_{n}}}=\frac{v_{n}^{2} r_{n}}{G M} \cong 1
$$

valid because of the virial theorem (15). The validity of the Formula (20) is checked for different planets in Table 1 and for satellites of Jupiter in Table 2.

By having the result (19) for $\Delta E$ we can apply it in the formula representing a quantum aspect of the classical Joule-Lenz law for the dissipation of energy [8]-[13]:

$$
\Delta E \Delta t=h \text {. }
$$

A substitution of $\Delta E$ from (19) into (21) yields

Table 1. Check of validity of the formula (20) done for the planets; the gravitational constant $G$ is taken from (10) and $M$ is the solar mass from (11); $v$ is the average planet velocity in $\mathrm{km} / \mathrm{sec}, r$ is the average distance between the planet and Sun in $10^{6} \mathrm{~km}$.

\begin{tabular}{ccccc}
\hline Planet & $V$ & $r$ & $V^{2} r\left(\right.$ in $\left.10^{20} \mathrm{~m}^{3} \cdot \mathrm{s}^{-2}\right)$ & $G M\left(\right.$ in $\left.10^{20} \mathrm{~m}^{3} \cdot \mathrm{s}^{-2}\right)$ \\
\hline Mercury & 47.89 & 57.91 & 1.328 & 1.327 \\
Venus & 35.03 & 108.20 & 1.328 & 1.327 \\
Earth & 29.79 & 149.60 & 1.328 & 1.327 \\
Mars & 24.13 & 227.94 & 1.327 & 1.327 \\
Jupiter & 13.06 & 778.33 & 1.328 & 1.327 \\
Saturn & 9.64 & 1426.98 & 1.326 & 1.327 \\
Uranus & 6.81 & 2870.99 & 1.331 & 1.327 \\
Neptune & 5.43 & 4497.07 & 1.326 & 1.327 \\
Pluto & 4.74 & 5913.52 & 1.329 & 1.327 \\
\hline
\end{tabular}


Table 2. Check of validity of the formula (20) done for the satellites of the Jupiter planet. $G$ is the same as that taken in Table 1 [see (10)] but the mass of Jupiter is $M_{J}=1.9 \times 10^{27} \mathrm{~kg}$ [3] [4]. The velocity $v$ is calculated according to the formula $2 \pi r / T$ where $r$ is the average distance of the satellite from the Jupiter center (in $\mathrm{km}$ ) and $T$ is the time period of the satellite circulation about the Jupiter planet expressed as a multiple of 86,400 seconds.

\begin{tabular}{|c|c|c|c|c|}
\hline Satellite & $r$ & $T$ & $v^{2} r\left(\right.$ in $\left.10^{16} \mathrm{~m}^{3} \cdot \mathrm{s}^{-2}\right)$ & $G M_{J}\left(\right.$ in $\left.10^{16} \mathrm{~m}^{3} \cdot \mathrm{s}^{-2}\right)$ \\
\hline Metis & 127,360 & 0.295 & 12.73 & 12.67 \\
\hline Adrastea & 128,980 & 0.298 & 12.78 & 12.67 \\
\hline Amaltea & 181,300 & 0.498 & 12.71 & 12.67 \\
\hline Tebe & 221,900 & 0.675 & 12.68 & 12.67 \\
\hline Io & 421,600 & 1.769 & 12.66 & 12.67 \\
\hline Europa & 670,900 & 3.551 & 12.66 & 12.67 \\
\hline Ganimedes & $1,070,000$ & 7.155 & 12.66 & 12.67 \\
\hline Callisto & $1,883,000$ & 16.689 & 12.68 & 12.67 \\
\hline Leda & $11,094,000$ & 238.72 & 12.67 & 12.67 \\
\hline Himalia & $11,480,000$ & 250.57 & 12.74 & 12.67 \\
\hline Lysithea & $11,720,000$ & 259.22 & 12.67 & 12.67 \\
\hline Elara & $11,737,000$ & 259.65 & 12.68 & 12.67 \\
\hline Ananke & $21,200,000$ & 631 & 12.66 & 12.67 \\
\hline Carme & $22,600,000$ & 692 & 12.75 & 12.67 \\
\hline Pasifae & $23,500,000$ & 735 & 12.70 & 12.67 \\
\hline Sinope & $23,700,000$ & 758 & 12.25 & 12.67 \\
\hline
\end{tabular}

from which

$$
\Delta t=\frac{2 \pi r_{n}}{v_{n}}=T=1 \text { year. }
$$

This holds because $2 \pi r_{n}$ is the approximate path length of the Earth about the Sun and $v_{n}$ is the average speed of the Earth planet. In effect-in view of (22) and (23)the energy $\Delta E$ by which the Earth planet energy is lowered is quite small:

$$
\Delta E=\frac{h}{T}=\frac{h}{365.26 \times 86400 \mathrm{~s}} \cong 2 \times 10^{-34} \mathrm{erg} .
$$

Results (23) and (24) are similar to those attained before in the quantum-theoretical calculations [8]-[13] where the Formula (21) is established. In effect of that formula, as well as (23), the transition time $\Delta t$ is equal to the circulation time period of a moving particle and the transition energy $\Delta E$ is equal to $h$ divided by that period of time.

\section{Discussion}

A peculiar result is that the quantum of energy (24) emitted by the Earth is much 
smaller than that emitted by a hydrogen atom for the excited states $n$ having the quantum numbers equal to about $n=10$. This is explained by the fact that for the emission energy $\Delta E$ from the quantum state $n+1$ to state $n$ of the atom we have

$$
\Delta E=\frac{h}{T_{n}}
$$

where

$$
T_{n}=\frac{n^{3} h^{3}}{4 \pi^{2} m e^{4}}=n^{3} \times 1.5 \times 10^{-16} \mathrm{~s} .
$$

This time is much shorter than $T=1$ year characteristic for the circulation time of the Earth, and $h$ entering the numerator of the Formula (25) does remain unchanged equally for the electron transition as well as for the transition of a planet from state $n+1$ to $n$. The Formula (25a) gives

$$
T_{n} \sim n^{3}
$$

therefore for a sufficiently large $n$ we can obtain

$$
T_{n}=1 \text { year }
$$

also for the hydrogen atomic state. In this case, however, the electron energy in the atom would be very close to zero giving a situation corresponding to the positive hydrogen ion.

All processes the effect of which can be completely annulled, are called reversible [14]. Evidently, the spontaneous emission of the kind of (24) makes irreversible the planetary, or satelitary, motion too.

\section{Conclusions}

In the framework of the classical mechanics a celestial body entering the solar system is circulating incessibly about its gravitational center without any loss of energy. In other words in the classical theory we cannot indicate a parameter existent in the system which will cause any slowdown of the motion.

A different situation is obtained in the scheme of the old quantum theory. Here a planet, say the Earth, is located on a very high quantum level, but the energy distant of that level from the nearest lower level is very small. It is not compulsory for a planet to make a step to this lower level, nevertheless such possibility does exist. According to the quantum aspect of the Joule-Lenz law the time period necessary for such step is rather long: for example for the Earth planet it is equal to one year. Next the situation of a planet circulating about the Sun is repeated, but now the planet energy is slightly smaller than before its first step. The second step can be done to the next lower energy level in course of the time period which is close to that necessary for the first step.

In effect the rate of decrease of the planet energy is very small. For the Earth it is about

$$
2 \times 10^{-34} \text { erg }
$$

per one year. 
Correspondingly to the lowering of energy, the distance between a planet and the Sun will be shortened.

A limiting situation will be attained when these two bodies-a planet and the Sun-will touch together. Such a picture is fully absent in the framework of the classical mechanics.

But beyond of the shortage of the distance between two masses-in virtue of the formula [2]

$$
\frac{T_{n}^{2}}{a_{n}^{3}}=\frac{4 \pi^{2}}{G M} \approx \frac{T_{n}^{2}}{r_{n}^{3}}
$$

-the time period $T_{n}$ of the planet circulation about the mass $M$ of the Sun will be shortened too: because of (29) we have

$$
T_{n} \sim r_{n}^{3 / 2},
$$

so the shortening of $T_{n}$ will be stronger than that of $r_{n}$.

On the other hand, in virtue of (30), the planet velocity about the Sun, i.e.

$$
v_{n} \cong \frac{2 \pi r_{n}}{T_{n}} \sim \frac{r_{n}}{r_{n}^{3 / 2}}=\frac{1}{r_{n}^{1 / 2}},
$$

will be slightly increased with a decrease of $r_{n}$.

In calculating the angular momentum

$$
L_{n}=m v_{n} r_{n}
$$

of a planet which for the state $n+1$ is proportional to $S$ in (2), the effects concerning $r_{n}$ and $v_{n}$ combine into

$$
L_{n+1} \sim n+1, \quad L_{n} \sim n, \quad L_{n-1} \sim n-1, \quad \text { etc. }
$$

It should be noted here that the effect of reduction of the mass $M$ of the Sun [see (11)] due to the energy emission has been fully neglected. This effect leads evidently to an increase of the energy of a moving planet, so it acts in direction opposite to the quantum decrease of energy presented in the paper. Physically this means that the energy decrease discussed above (Section 3 and Section 4) is more sound for the satellites of non-radiating planets than for the planets themselves.

\section{References}

[1] Sommerfeld, A. (1931) Atombau und Spektrallinien. Vol. 1. 5th Edition, Vieweg, Braunschweig.

[2] Sommerfeld, A. (1943) Mechanik, Akademische Verlagsgesellschaft, Leipzig.

[3] Lang, K.L. (1991) Astrophysical Data: Planets and Stars. Springer, New York.

[4] Królikowska-Sołtan, M., Kwast, T., Sołtan, A. and Sroczyńska-Kożuchowska, M. (1994) Astronomy-School Dictionary (in Polish). Wydawnictwa Szkolne i Pedagogiczne, Warszawa.

[5] Blanco, V.M. and McCuskey, S.W. (1961) Basic Physics of the Solar System. AddisonWesley, Reading.

[6] Becker, R.A. (1954) Introduction to Theoretical Mechanics. McGraw-Hill, New York. 
[7] Landau, L.D. and Lifshits, E.M. (2002) Theoretical Physics. Vol. 1. Mechanics (in Russian). 5th Edition, Fizmatlit, Moscow.

[8] Olszewski, S. (2015) Journal of Modern Physics, 6, 1277-1288. http://dx.doi.org/10.4236/jmp.2015.69133

[9] Olszewski, S. (2016) Reviews in Theoretical Science, 4, 336-352. http://dx.doi.org/10.1166/rits.2016.1066

[10] Olszewski, S. (2016) Journal of Modern Physics, 7, 162-174. http://dx.doi.org/10.4236/jmp.2016.71018

[11] Olszewski, S. (2016) Journal of Modern Physics, 7, 827-851. http://dx.doi.org/10.4236/jmp.2016.78076

[12] Olszewski, S. (2016) Journal of Modern Physics, 7, 1440-1448. http://dx.doi.org/10.4236/jmp.2016.712131

[13] Olszewski, S. (2016) Quantum Matter, 5, 664-669. http://dx.doi.org/10.1166/qm.2016.1360

[14] Lindsay, R.B. and Margenau, H. (1963) Foundations of Physics. Dover Publications, New York.

Submit or recommend next manuscript to SCIRP and we will provide best service for you:

Accepting pre-submission inquiries through Email, Facebook, LinkedIn, Twitter, etc. A wide selection of journals (inclusive of 9 subjects, more than 200 journals)

Providing 24-hour high-quality service

User-friendly online submission system

Fair and swift peer-review system

Efficient typesetting and proofreading procedure

Display of the result of downloads and visits, as well as the number of cited articles Maximum dissemination of your research work

Submit your manuscript at: http://papersubmission.scirp.org/

Or contact jmp@scirp.org 